\title{
Philosophieren mit Kindern als Pädagogik für eine offene und ungewisse Zukunft
}

\author{
Kerstin Michalik \\ Universität Hamburg
}

Kontakt: kerstin.michalik@uni-hamburg.de

eingereicht: 04.06.2021; überarbeitet: 07.09.2021; angenommen: 10.09.2021

\begin{abstract}
Zusammenfassung: Welchen Beitrag kann das Philosophieren mit Kindern dazu leisten, Kinder auf eine Welt vorzubereiten, die durch rapiden Wandel, eine Zunahme von Ungewissheiten und insbesondere durch eine immer bedrohlicher erscheinende ökologische Krise gekennzeichnet ist? Die Frage, wie Bildungs- und Lernprozesse im Hinblick auf eine offene und ungewisse, immer weniger vorhersehbare Zukunft gestaltet und wie Kinder darauf vorbereitet werden können, mit Ungewissheit und Komplexität konstruktiv umzugehen, wird in der Erziehungswissenschaft seit längerer Zeit in verschiedenen Kontexten diskutiert. Es sind „Zukunfts-“ oder auch „Ungewissheitskompetenzen" definiert worden, es gibt jedoch bislang wenig konkrete Vorstellungen, wie solche Kompetenzen bei Kindern und Jugendlichen entwickelt werden können. Und angesichts des Umstandes, dass eine ungewisse Zukunft nicht vorhersehbar ist, werden auch Erziehungsziele und -prozesse als solche zunehmend ungewiss und fraglich.
\end{abstract}

Schlagwörter: Ungewissheit, Philosophieren mit Kindern, Ökologische Krise, Bildung für nachhaltige Entwicklung

Abstract: This paper explores the question of whether and how philosophizing with children can help to prepare them for a world of rapid change and growing uncertainty in an era of risk, and in particular with regard to the problems of sustainability. There is a growing concern in educational science that, in a world that is changing rapidly, it is essential to prepare children to deal with uncertainty, ambiguity and complexity in a range of contexts. Our globalised world is increasingly perceived as a 'post-normal' era characterized by high and growing levels of complexity, the questioning of old certainties, contested scientific knowledge and democratic values, and recently also by a new worldwide. Special skills and "future" or "uncertainty competencies" have been designed to prepare children for an unknown and open future, but experts generally offer limited guidance as to how such competencies can be achieved. And given that an uncertain future is by definition hard to predict, educational aims and processes are in themselves increasingly uncertain and need to be questioned.

Keywords: Uncertainty, Philosophizing with Children, Ecological Crisis, Education for Sustainable Development

Welchen Beitrag kann das Philosophieren mit Kindern dazu leisten, Kinder auf eine Welt vorzubereiten, die durch rapiden Wandel, eine Zunahme von Ungewissheiten und insbesondere durch eine immer bedrohlicher erscheinende ökologische Krise gekennzeichnet ist? Unsere heutige globalisierte Welt wird zunehmend als eine „post-normale“ Zeit (Tauritz, 2012, S. 299) oder als ein „Zeitalter von Ungewissheit" (Wals, 2010, S. 145) wahrgenommen, das sich durch ein hohes Maß an Komplexität, durch die Infragestellung alter Gewissheiten und zunehmend auch wissenschaftlicher Erkenntnisse und demokratischer Werte auszeichnet.

Diese Entwicklungen stellen das Bildungssystem vor neue und besondere Herausforderungen. Die Frage, wie Bildungs- und Lernprozesse im Hinblick auf eine offene und ungewisse, immer weniger vorhersehbare Zukunft gestaltet und wie Kinder darauf vorbereitet werden können, mit Ungewissheit und 
Komplexität konstruktiv umzugehen, wird in der Erziehungswissenschaft seit längerer Zeit in verschiedenen Kontexten (Barnett, 2000; Barnett, 2012; Helsper, Hörster \& Kade, 2003; Hall, 2006; Hayden, 2012; Fecho, 2013; D'Agnese, 2018; Paseka, Keller-Schneider \& Combe, 2018) diskutiert. In diesen Diskussionszusammenhängen sind „Zukunfts-“ oder auch „Ungewissheitskompetenzen“ definiert worden, die dazu beitragen sollen, Kinder und Jugendliche auf neue und ungewisse Herausforderungen vorzubereiten, es gibt jedoch bislang wenig konkrete Vorstellungen, wie solche Kompetenzen bei Kindern und Jugendlichen entwickelt werden können. Und angesichts des Umstandes, dass eine ungewisse Zukunft nicht vorhersehbar ist, werden auch Erziehungsziele und -prozesse als solche zunehmend ungewiss und fraglich.

Das Philosophieren mit Kindern als ein Unterrichtsprinzip in allen Fächern und Schulstufen (Michalik, 2013; Michalik, 2016) kann in verschiedener Hinsicht einen wichtigen Beitrag leisten, um auf die Herausforderungen einer zunehmend ungewissen Welt vorzubereiten. Ungewissheit ist ein zentrales Moment des Philosophierens mit Kindern, weil es auf philosophische Fragen keine richtigen oder falschen Antworten, sondern immer nur verschiedene Antwortmöglichkeiten gibt. Das Philosophieren zielt darauf ab, kritisches, kreatives, kooperatives Denken und vor allem auch ethische Urteilskraft zu entwickeln, die für einen konstruktiven Umgang mit Ungewissheit besonders bedeutsam ist. Als eine kritische Pädagogik kann das Philosophieren mit Kindern spezifischen Problemen und Spannungsfeldern von Erziehungsprozessen begegnen, die darauf abzielen, Kinder auf eine Zukunft vorzubereiten, die wir weder vorhersehen noch vorbestimmen können und dürfen.

\section{Bildung für eine ungewisse Zukunft - Herausforderungen für das Lehren und Lernen}

Die Herausforderungen einer zunehmend durch Komplexität und Ungewissheit gekennzeichneten, durch Klimawandel, Naturzerstörung, politische und ökonomische Krisen bedrohten Welt für Bildung und Erziehung in Gegenwart und Zukunft werden unter anderem im Rahmen der Bildung für nachhaltige Entwicklung (Education for Sustainable Development) in den letzten zwei Jahrzehnten international diskutiert (Gordon, 2006; Wals \& Corcoran, 2012; Lambrechts \& Hindson, 2016; Tauritz, 2012, 2016, 2019). In diesem Kontext werden nicht nur neue Erziehungsziele und -inhalte und neue LehrLernformen (Hall, 2006; Barnett, 2012; Fecho, 2013), sondern ein radikaler Wandel (Tauritz, 2016, S. 101) des gesamten Bildungssystems gefordert. Im Mittelpunkt steht die Frage, welche Kompetenzen, Fähigkeiten und Fertigkeiten, Einstellungen und Haltungen erforderlich sind, um gegenwärtigen und zukünftigen globalen Herausforderungen konstruktiv begegnen und im Sinne eines demokratischen und friedlichen Zusammenlebens handlungsfähig bleiben zu können.

Als eine zentrale Kernkompetenz gilt die Fähigkeit, mit einer ungewissen Faktenlage, „Knowledge Uncertainty", umgehen zu können, im Sinne der Entwicklung und Förderung von Ungewissheitskompetenzen, definiert als „specific set of skills, knowledge, attitudes and capabilities needed to deal with uncertainty, ambiguity and complexity in diverse contexts" (Tauritz, 2016, S. 90, S. 93). Konkret geht es hier zum Beispiel darum, Ungewissheit als Katalysator für kreative Handlungen nutzen zu können, um Empathiefähigkeit und einen konstruktiven Umgang mit verschiedenen Perspektiven, um eine forschende Haltung und um die Bereitschaft, eigenes Denken zu reflektieren und eigene Vorstellungen zu verändern (Tauritz, 2012, S. 307; Tauritz 2016, S. 94). Im Rahmen der Bildung für Nachhaltige Entwicklung sind in Anlehnung an das Konzept der OECD-Schlüsselkompetenzen (OECD, 2005) weitere zentrale, auf Handlungsfähigkeit abzielende „Gestaltungskompetenzen“ formuliert worden (de Haan et al., 2008, S. 188, S. 237):

- Kompetenz zum Umgang mit Ambivalenzen und Uneindeutigkeiten als Grundlage für das Handeln und Entscheiden angesichts unvollständiger und überkomplexer Informationen

- Dilemmakompetenz zur Bewältigung individueller und kollektiver Entscheidungsdilemmata, basierend auf Ambiguitätstoleranz (Fähigkeit, Entscheidungsprozesse auf der Grundlage sich wider- 
sprechender Informationen zu gestalten) und Ambivalenztoleranz (emotionale Bereitschaft, widersprüchliche Problemdefinitionen zuzulassen)

- Kompetenz zum moralischen Handeln auf der Grundlage von Vorstellungen zu Gerechtigkeit

Ungewissheits- oder Gestaltungskompetenzen, wie sie im Kontext der Bildung für Nachhaltige Entwicklung und der "climate change education“ diskutiert werden, sind auch im Hinblick auf den Umgang mit neuen Informations- und Kommunikationstechnologien und Künstlicher Intelligenz und insbesondere auch für die Demokratiebildung relevant. Die Zunahme populistischer Bewegungen in Europa kann auch als eine Form der Komplexitätsreduktion, als Reaktion auf zunehmende Ungewissheiten sowohl bezogen auf die eigene individuelle Situation als auch globale Entwicklungen gedeutet werden.

Die Fähigkeit zum Umgang mit Ungewissheit ist zu einem Kernelement der Diskussion um eine zukunftsfähige Bildung geworden und es besteht ein zunehmender Konsens darüber, dass dies tiefgreifende Implikationen für das schulische Lehren und Lernen und die Lehrplanentwicklung haben muss, weil das bisherige Erziehungssystem wenig geeignet ist, um solche Kompetenzen und Bildungsinhalte zu vermitteln. Denn das Ziel, Ungewissheit und den Umgang damit im Rahmen von Bildungsprozessen zu berücksichtigen, steht in einem klaren Spannungsverhältnis zur „Kultur der Gewissheit“ (Rowley, 2004, S. 88), die das bestehende Schulsystem prägt. Nachhaltigkeits- und Klima-Bildungsziele sind zwar seit der weltweiten OECD-Bildungsoffensive 2009-2014 in vielen Ländern in die Curricula integriert worden, die Schulsysteme basieren jedoch nach wir vor und seit PISA in einem zunehmenden Ausmaß auf (scheinbaren) Gewissheiten (Gordon, 2006): Reduktion von inhaltlicher Offenheit und Komplexität im Hinblick auf Wissensreproduktion zum Zweck von Leistungsmessung und -bewertung, standardisierte Curricula und standardisierte Kompetenzformulierungen, Output-Orientierung und evidenzbasierte Lehr-Lernarrangements, individuelle anstelle von kooperativen Lernprozessen.

Um Komplexität und Ungewissheit als wesentliche Komponenten des Lehrens und Lernens in Erziehungs- und Bildungsprozesse zu integrieren, sind andere Lernumgebungen erforderlich, die von verschiedenen Autor*innen wie folgt charakterisiert werden: Lernumgebungen, die emotionale Sicherheit bieten, in denen verschiedene Sichtweisen und Deutungen ohne Bewertung und Benotung offen und frei diskutiert werden können, in denen die Schüler*innen akzeptieren können, dass es zu den meisten komplexen Problemen keine einfachen und klaren Antworten gibt, prozessorientierte, ergebnisoffene Formen von Unterricht, in denen die gemeinsame Sinn- und Bedeutungsfindung und eine reflexive Praxis im Mittelpunkt stehen, interdisziplinäre Themen und komplexe Problemstellungen, flexible Rollen von Lehrenden und Lernenden und Lehrkräfte, die sich nicht primär als Wissensvermittler*innen, sondern als Expert*innen für Nichtwissen und Ungewissheit verstehen (Gordon, 2006; Barnett, 2012; Hall, 2006, Davies, 2012; Tauritz, 2012; Tauritz, 2016; Tauritz, 2019; Marcussen, Weiss \& Helskog, 2021; Kavanagh, Waldron \& Mallon, 2021; Waldron, Mallon \& Kavanagh, 2021). Dabei besteht weitgehend Einigkeit darüber, dass es bei der Vorbereitung von Kindern auf einen angstfreien und konstruktiven Umgang mit Ungewissheit nicht darum gehen kann, isolierte Kompetenzen zu vermitteln, sondern dass es sich hierbei um einen interdisziplinären, ganzheitlichen Zugang handeln muss, der das gesamte Curriculum erfasst und auf einem grundlegenden Wandel bisheriger pädagogischer Paradigmen basiert.

Erfordernisse für das Schulsystem sind damit zwar benannt, es gibt jedoch noch wenig Forschung zu konkreten Unterrichtskonzepten, die dazu beitragen können, Kinder und Jugendliche auf den Umgang mit Ungewissheit und Komplexität vorzubereiten (Hall, 2006; Jordan \& McDaniel, 2014; Beghetto, 2017; Tauritz, 2019, Davies, 2012). Und es gibt auch noch wenige Erkenntnisse darüber, wie Lehrkräfte im Hinblick auf so eine weitreichende Veränderung ihrer Rolle und ihres Selbstverständnisses als Lehrkraft aus- und fortgebildet werden können (Tauritz, 2016, S. 101). 
In den Diskussionszusammenhängen um neue und alternative Bildungskonzepte für die Zukunft hat das Philosophieren mit Kindern als Gesprächsmethode, Unterrichtsprinzip und pädagogische Haltung bislang nur begrenzt Aufmerksamkeit erhalten, obwohl die Bedeutung und die Potenziale dieses Ansatzes für die Umwelt- und Nachhaltigkeitsbildung von Seiten der Vertreter*innen der Kinderphilosophie bereits seit geraumer Zeit diskutiert worden sind (Rowley \& Lewis, 2003; Rowley, 2004; Müller, 2011; Künzli David, Buchs, Wüst, 2015; MacDonald \& Bowen, 2016; MacDonald, Bowen und Hill, 2017; Lyle, 2018; Prust \& Sukopp, 2017; Sukopp, 2020; Birch, 2020; Marcussen et al., 2021). Ungewissheit ist in der Tat ein Schlüsselelement des Philosophierens mit Kindern, und der Prozess des gemeinsamen Nachdenkens ist eine Spielwiese für Erfahrungen mit Ungewissheit - philosophischer Ungewissheit - sowohl für Schüler*innen als auch für Lehrkräfte.

\section{Philosophisches Fragen und Forschen im Raum des Ungewissen}

In der philosophischen Forschungsgemeinschaft beschäftigen Kinder und Jugendliche sich mit philosophischen Fragen wie zum Beispiel: Können Pflanzen glücklich sein? Was ist Freundschaft? Was heißt es, fair zu sein? oder Woher kommt der Mensch? Es geht um Fragen und komplexe Inhalte, die offen sind für verschiedene Interpretationen und Weltdeutungen. Beim Philosophieren mit Kindern steht der Umgang mit Ungewissheit im Zentrum des gemeinsamen Nachdenkens, es gibt keine gewissen Antworten und auch keinen zwingend erforderlichen Konsens am Ende der Gespräche. Das Philosophieren kann mit kontroversen Positionen und offenen Fragen enden. Die Ergebnisse sind ebenso ungewiss wie der Prozess des Philosophierens und die Richtungen, die das Gespräch nimmt. Im philosophischen Gespräch müssen sowohl Schüler*innen als auch Lehrkräfte mit Ungewissheit umgehen.

Lernen und Bildung für eine ungewisse Zukunft bezieht sich nicht nur auf den Umgang mit Nicht-Wissen angesichts komplexer Zusammenhänge und Wirkungsgefüge in einer sich schnell verändernden, globalisierten und vernetzen Welt. Es geht auch und insbesondere um grundsätzliche erkenntnistheoretische Fragen und Zusammenhänge und um die grundsätzlichen Grenzen unserer Erkenntnismöglichkeiten als menschliche Wesen mit einem spezifischen und notwendig begrenzten Zugang zur Welt. Diese fundamentale, existentielle Form von Ungewissheit ist expliziter Gegenstand des Philosophierens: Was können wir wissen? Was dürfen wir hoffen? Was sollen wir tun? Was ist der Mensch? - mit diesen Grundfragerichtungen lassen sich nach Kant die Inhalte der Philosophie beschreiben (Kant, 1968) und diese sind auch Gegenstand des Philosophierens mit Kindern und zentral für das Nachdenken darüber, wie wir jetzt und in Zukunft auf diesem Planeten leben wollen.

Das Philosophieren mit Kindern zeichnet sich durch weitere Merkmale aus, die auf Ungewissheit bezogen und unmittelbar relevant für eine Bildung für die Zukunft sind. Als Methode des Austausches und der Weiterentwicklung von Gedanken zielt es darauf ab, kritisches, kooperatives, kreatives und verantwortungsvolles Denken zu fördern (Lipman, Sharp \& Oscanyan, 1980; Lipman, 2003). Kinder und Jugendliche lernen, Dinge kritisch zu hinterfragen, eigene Meinungen und Positionen zu begründen, Argumente zu entwickeln und zu prüfen, deren Voraussetzungen und Folgen zu hinterfragen. In der philosophischen Forschungsgemeinschaft steht das gemeinsame Denken im Mittelpunkt, es geht um den Austausch von Gedanken, das kollektive Konstruieren von Bedeutungen, das Aufnehmen anderer und neuer Perspektiven, es geht um Selbstreflexion und den Wechsel von Meinungen im Lichte neuer Argumente. Damit wird auch kreatives Denken gefördert, denn im offenen Raum des Philosophierens gibt es keine Grenzen für das Entwickeln neuer Gedanken. Die philosophische Forschungsgemeinschaft ist konzipiert als ein ",safe place”, sie setzt Respekt und Wertschätzung der Teilnehmer*innen untereinander voraus. Emotionale Sicherheit, das heißt die Gewissheit, dass alle Stimmen wichtig sind und gehört werden und jeder Beitrag ernstgenommen wird, ist eine wichtige Voraussetzung für einen angstfreien Umgang mit Ungewissheit.

Das philosophische Gespräch ist ein Lernsetting, das sich von herkömmlichen, gewissheitsbasierten 
Unterrichtszusammenhängen deutlich unterscheidet und in dieser Hinsicht auch als eine „counter-cultural-practice" (O'Riordan, 2016, S. 658) bezeichnet worden ist. Das gemeinsame Nachdenken über offene Fragen hat Auswirkungen auf das Interaktions- und Beziehungsgefüge zwischen Lehrkräften und Schüler*innen. Im philosophischen Gespräch ist die Lehrkraft nicht mehr die Vermittlerin von Wissen, denn philosophische Fragen sind auch für die Lehrkraft offen. Im philosophischen Gespräch übt sich die Lehrkraft auf der inhaltlichen Ebene in Zurückhaltung. Ihre wichtigste Aufgabe besteht darin, den Prozess des Nachdenkens durch geeignete Impulse zu vertiefen, indem die Kinder zum Beispiel dazu angeregt werden, sich aufeinander zu beziehen, Meinungen zu begründen, Argumente zu prüfen, Beispiele und Gegenbeispiele zu finden (Michalik \& Schreier, 2017, S. 100). Die Ungewissheit und Unvorhersehbarkeit der Dynamik und der Ergebnisse philosophischer Gespräche verändert das klassische Rollengefüge und damit auch das Machtgefälle zwischen Lehrenden und Lernenden. Philosophische Gespräche bieten damit Raum für intensive, bedeutungsvolle und authentische Gespräche und eine Begegnung von Erwachsenen und Kindern und Jugendlichen auf Augenhöhe (Murris, 2008; Scholl, Nichols \& Burgh, 2014; Michalik, 2019).

Das Philosophieren mit Kindern wurde von seinen Begründer*innen Lipman und Sharp als eine „kritische und reflexive pädagogische Praxis“ (Lipman, 2003, S. 18) verstanden, die sich von herkömmlichen Erziehungsparadigmen deutlich unterschied, und zwar im Hinblick auf das Wesen und die Ziele von Erziehung und Bildung, die Rolle der Lehrkräfte im Erziehungsprozess und auch den Stellenwert von Wissen an sich. In Lipmans „kritischer pädagogischer Praxis“ besteht Bildung nicht in der Vermittlung von Wissen durch eine Lehrkraft an nichtwissenden Schüler*innen, sondern Erziehung besteht in der Teilnahme an einer von den Lehrkräften geleiteten Forschungsgemeinschaft, in der es um Verstehen, um das Herstellen von Beziehungen und um die Entwicklung einer eigenständigen Urteilskraft geht. Wissen ist in erkenntniskritscher Hinsicht nicht eindeutig und gewiss und in klar voneinander abgrenzbare Disziplinen aufteilbar, sondern mehrdeutig, vorläufig und ungewiss. Die Lehrkraft ist folglich auch keine Autorität, die eindeutige Gewissheiten vermittelt, sondern sie ist Teil eines gemeinsamen Forschungs- und Erkenntnisprozesses und als solche durchaus auch fehlbar (Lipman, 2003, S. 18). In der zweiten Generation der Kinderphilosoph*innen hat sich das Augenmerk verstärkt auf die Infragestellung der Machtstrukturen zwischen Erwachsenen und Kindern und im schulischen Kontext von Lehrkräften und Schüler*innen gerichtet. Als eine kritische Pädagogik geht es hier darum, bestehende Konzeptualisierungen von „Kind“ zu hinterfragen und den Stimmen der Kinder als ernst zu nehmende und gleichgewichtige Beiträge im Prozess des gemeinsamen Verstehens und der gemeinsamen Sinnbildung Gehör zu verschaffen (Haynes, 2016). ${ }^{1}$

In der Diskussion um das Erfordernis neuer und alternativer Lernformen für eine Bildung für nachhaltige Entwicklung ist argumentiert worden, dass die für diese Thematik relevanten Werte und (Ungewissheits-)Kompetenzen nicht im Rahmen einer traditionellen „Top-down”-Didaktik vermittelt werden können. Gefragt seien stattdessen neue Formen der Beziehung zwischen Lehrkräften und Schüler*innen im Sinne dialogischer Lern- und Interaktionsformen und im Sinne einer Kultivierung des Nicht-Wissens von Lehrenden und Lernenden (Marcussen et al., 2021, S. 18; Tauritz, 2016, S. 101). Das Philosophieren bietet den Raum für solche neue Formen der Begegnung und der Interaktion zwischen Lehrenden und Lernenden und sollte daher in das gesamte Curriculum integriert werden, nicht zuletzt auch aufgrund der besonderen Bedeutung ethischer Reflexions- und Urteilsfähigkeit im Kontext einer Bildung für die Zukunft.

\footnotetext{
${ }^{1}$ In dieser Hinsicht sind auch Bezüge hergestellt worden zur kritischen Pädagogik Paulo Freires, nach der das Ziel von Erziehung und Bildung die menschliche Befreiung im Sinne der Förderung sozialer Gerechtigkeit und der Aufhebung von gesellschaftlicher Unterdrückung ist (Funston, 2017; Kohan, 2018).
} 


\section{Ethische Urteilsbildung}

Die Entwicklung sozialen und ethisch verantwortlichen Denkens ist ein weiteres wichtiges Ziel des Philosophierens mit Kindern (Sharp, 2018; Morehouse, 2018) und für die Urteilsbildung in komplexen Entscheidungsprozessen unmittelbar relevant (Lyle, 2018). In Zeiten globaler Krisen und besonderer Herausforderungen für die Demokratie haben ethische Fragen eine große Bedeutung und sollten fächerübergreifend Berücksichtigung finden. Wie wir die Zukunft gestalten wollen und können, ist im Hinblick auf die ökologische Krise und die damit verbundenen ökonomischen, ökologischen, sozialen und kulturellen Herausforderungen mit vielen normativen Fragen verbunden, die der ethischen Reflexion bedürfen. Hier geht es zum Beispiel um das Verhältnis zwischen Mensch und Natur, Fragen der intergenerationalen, intragenerationalen wie auch internationalen Gerechtigkeit und Fairness, der Verantwortung für gegenwärtige und zukünftige Generationen sowie Fragen der ökonomischen Entwicklung und des Konsums, die in der Philosophie als Fachdisziplin zum Beispiel im Rahmen von Ökologischer Ethik, Umwelt- und Klimaethik thematisiert werden (Reder, Gösele, Köhler \& Wallacher, 2019; Birnbacher, 2016; Roser \& Seidel, 2015; Ott, 2010).

Im Rahmen der Bildung für Nachhaltige Entwicklung ist die zentrale Bedeutung ethischer Urteilsfähigkeit, der Fähigkeit, ethische Normen und Konzepte zu reflektieren, als eine wichtige Bedingung für Handlungsfähigkeit formuliert worden. Es geht es um den Umgang mit individuellen und kollektiven Entscheidungsdilemmata im Sinne von „Dilemmakompetenz", um Entscheidungsfähigkeit unter der Bedingung von Ungewissheit und allgemein um die Fähigkeit zum moralischen Handeln auf der Basis von Gerechtigkeitsvorstellungen (de Haan et al., 2008; Künzli David et al., 2015). Auf der inhaltlichen Ebene kann das Philosophieren mit Kindern und Jugendlichen eine Grundlage für die Reflexion zentraler Begriffe wie Gerechtigkeit sein (Kavanagh et al., 2021). Als komplexer philosophischer Begriff kann Gerechtigkeit nicht voraussetzungslos zum Kriterium für die Bewertung von Problem- und Konfliktsituationen gemacht werden, sondern es bedarf spezifischer Lernarrangements, die auf eine philosophische Begriffsklärung und die Förderung eigener ethischer Urteilskompetenz abzielen: Was ist Gerechtigkeit? Was bedeutet Gerechtigkeit für gegenwärtige und zukünftige Generationen und hat der Begriff vielleicht verschiedene Bedeutungen in unterschiedlichen kulturellen Kontexten? Solche Bedeutungen müssen ausgehandelt werden und setzen die Fähigkeit zu interkultureller Kommunikation und zum Perspektivenwechsel sowie zum kritischen Hinterfragen eigener, kulturell bedingter Werte und Normen voraus.

In einer demokratischen, pluralistischen Gesellschaft können Werte und Normen nicht einfach vorgegeben werden, sondern müssen zum Gegenstand kommunikativer Prozesse gemacht werden (Tiedemann, 2007; Weber, 2013; Gebhard \& Michalik, 2017). Das Paradox der Wertevermittlung in einer pluralistischen Gesellschaft ist nur aufhebbar, wenn man den offenen Dialog über Werte selbst als Voraussetzung begreift: „Die pädagogische Situation ist nicht nur Vermittlungsort, sondern konstituiert selbst eine solche Wertekultur, d. h. ist Ort der Aneignung und Transformation von Werten" (Weber, 2013, S. 20). Sowohl zentrale Begriffe und Konzepte als auch Werte und Normen können in philosophischen Gesprächen hinterfragt, diskutiert, geklärt und mit Sinn und Bedeutung versehen werden.

Ein solcher offener Prozess des Umgangs mit Normen und Werten ist auch im Hinblick auf spezifische Probleme und Dilemmata, die im pädagogischen Diskurs zur Nachhaltigkeitsbildung diskutiert werden, relevant. Es ist kritisiert worden, dass mit der Schwerpunktverlagerung von der Umweltbildung und -erziehung zum Konzept der Bildung für Nachhaltige Entwicklung (UNESCO Dekade, 2005-2014, UNESCO, 2005) eine einseitig anthropozentrische und ökonomiezentrierte Ethik in den Vordergrund gerückt sei (Kopnina, 2012, 2014, 2020; Kopnina \& Cherniak, 2015; Kopnina \& Meijers, 2012). Während im Rahmen der Umweltbildung der 70er- und 80er-Jahre des 20. Jahrhunderts im Gefolge der Bewusstwerdung über die "Grenzen des Wachstums” (Meadows, Meadows, Randers \& Behrens III, 1972) ein ökologisches Basisverständnis und eine Sensibilisierung für das Erfordernis von Naturschutz zentral 
gewesen seien, habe sich mit den „Sustainable Development Goals“ der UNESCO (UNESCO, 2017) die Betonung sozialer und ökonomischer Faktoren in den Vordergrund geschoben. Dies beruhe auf der Unterstellung, dass die dreifache Zielsetzung einer sozialen, ökonomischen und ökologisch nachhaltigen Entwicklung in erster Linie durch ökonomisches Wachstum und ökonomische Entwicklung zu erreichen sei (Kopnina, 2020, S. 1.).

Diese Prioritätensetzung führe zu einem spezifischen Dilemma, weil Kritiker*innen der Nachhaltigkeitsziele davon ausgehen, dass ökonomisches Wachstum und industrielle Entwicklung und die damit verbundene Vermehrung von Bevölkerung und Konsum die zentrale Wurzel der Nachhaltigkeitsproblematik sind (Kopnina, 2020, S. 2). Angesichts solcher, den Nachhaltigkeitszielen innewohnenden Dilemmata erhalten Forderungen nach alternativen Formen einer „ökozentristischen Erziehung” oder auch „Ökopädagogik“ (Kahn, 2010), die darauf abzielen, ein inklusives planetarisches Bewusstsein zu entwickeln, in dessen Rahmen das Zusammenwirken und Teilen mit anderen auch nichtmenschlichen Lebewesen im Mittelpunkt steht, eine neue Aktualität (Kopnina, 2020, S. 5).

Im Kontext dieser Problemdiskussion kann das Philosophieren mit Kindern und Jugendlichen eine geeignete Plattform für kritisches Lernen bilden, das die Pluralität möglicher Perspektiven - anthropozentrisch, biozentrisch, holistisch - und das Hinterfragen aktueller Paradigmen, die das Verhältnis von Mensch und Natur oder existierender Formen des Konsums betreffen, zum Gegenstand philosophischen Nachdenkens macht:

- Was ist Natur? Ist der Mensch Natur? Haben nicht-menschliche Lebewesen wie Pflanzen und Tiere Rechte? Sind menschliche Bedürfnisse wichtiger als die anderer Lebewesen?

- Braucht der Mensch die Natur oder die Natur den Menschen?

- Haben Arten ein Recht auf Fortbestand? Sind wir verantwortlich für das Überleben und den Erhalt von Tieren und Pflanzen?

- Was brauchen Menschen zum Leben? Sind menschliche Bedürfnisse wichtiger als die anderer Lebewesen? Was ist wichtig im Leben?

Es handelt sich hier um Fragehorizonte (MacDonald \& Bowen, 2016; MacDonald, Bowen \& Hill, 2017; Müller, 2011; Michalik \& Schreier, 2017; Brüning, 2021; Brüning \& Nachtsheim, 2021) mit offenem Ausgang und der Möglichkeit, alternative Vorstellungen zu entwickeln. Denn vielleicht könnte es tatsächlich wichtig und notwendig sein, sich zur Lösung der immer drängender werdenden Umwelt- und Verteilungsprobleme auf der Welt auf ganz neue Denkpfade zu begeben und sowohl vorherrschende anthropozentrische Sichtweisen als auch etablierte Lebens-, Wirtschafts- und Konsummuster grundsätzlich in Frage zu stellen.

Das Philosophieren kann dazu beitragen die ethische Reflexions- und Urteilsfähigkeit von Kindern und Jugendlichen zu fördern, ohne vorgefertigte moralische Werte und Einstellungen zu vermitteln. Diese grundsätzliche Offenheit philosophischen Fragens und Forschens ist auch im Hinblick auf ein weiteres Spannungsfeld, das im Kontext der Nachhaltigkeitsbildung kontrovers diskutiert wird, von Bedeutung. Hier geht es um das Problem, dass es einerseits notwendig scheint, angesichts der drängenden ökologischen Probleme, bestimmte, auf eine nachhaltige Entwicklung bezogene Erziehungsziele konkret vorzugeben und andererseits demokratische und pluralistisch orientierte Lernprozesse für eine ungewisse und offene Zukunft zu gewährleisten.

\section{Lernen für eine offene und ungewisse Zukunft?!}

Wie offen ist die Zukunft und wie offen müssen Bildungsprozesse sein? Kontroversen gibt es nicht nur zu Zielen und damit verbundenen ethischen Ausrichtungen der Nachhaltigkeitsbildung, sondern auch im Hinblick auf die bildungsphilosophische Frage, wie Bildung und Erziehung gestaltet werden können im Hinblick auf eine Zukunft, die als zunehmend ungewiss gilt.

Die Rolle von Erziehung gilt es kritisch zu hinterfragen, auch im Licht der „Fridays for Future“-Bewe- 
gung, in der Kinder und Jugendliche ihre Besorgnisse und auch ihre Wut zum Ausdruck bringen angesichts der ökologischen Krise und des Fortbestands nicht-nachhaltiger Praktiken in Wirtschaft und Gesellschaft. Es ist zu hinterfragen, auf welcher moralischen Basis Erziehungsziele für spätere Generationen formuliert werden können.

Im Kontext der Bildung für nachhaltige Entwicklung wird international kontrovers diskutiert, vor welchen Herausforderungen das demokratische öffentliche Schulwesen im Hinblick auf die Vermittlung bestimmter, angesichts der ökologischen Krise als notwendig erachteter Bildungsziele konfrontiert ist: "On the one hand, there is a deep concern about the state of the planet and a sense of urgency that demands a break within existing non-sustainable systems, lifestyles, and routines, while on the other hand there is the conviction that it is wrong to persuade, influence, or even educate people towards pre- and expert-determined ways of thinking and acting" (Wals, 2010, S. 150). Während einerseits argumentiert wird, dass die Rückkehr zu einer „instrumentalistischen” Auffassung von Bildung und Erziehung erforderlich sei, weil die ökologischen Probleme gravierend und dringlich seien und ökologisch denkende Menschen zu deren Lösung beitragen könnten (Kopnina, 2012; 2020), wird andererseits davor gewarnt, dass Erziehung nicht als ein politisches Instrument für eine zukünftige, bereits vorbestimmte Gesellschafts- und Lebensform eingesetzt werden dürfe. Bildung für nachhaltige Entwicklung als eine normative Form von Bildung mit dem Ziel, eine nachhaltige Transformation der Gesellschaft auf der Basis spezifischer Entwicklungsziele und damit verbundener Werte, Einstellungen oder Kompetenzen zu erreichen, steht in Konflikt zu demokratischen, pluralistischen und emanzipatorischen Bildungsvorstellungen (Öhman, 2007; de Haan et al., 2008; Tauritz, 2016; Holfelder, 2019). Solche klar definierten Erziehungsziele stehen auch in einem unübersehbaren Spannungsverhältnis zu der allgemeinen Einschätzung, dass Zukunft hochgradig kontingent, nicht-linear, ungewiss und unvorhersehbar geworden ist.

Nach Holfelder impliziert die Kampagne für eine Bildung für Nachhaltige Entwicklung, dass eine nachhaltige Zukunft erreicht werden könne, wenn Menschen entsprechend erzogen würden, und dass Zukunft etwas sei, das geformt werden könne. Sie argumentiert, dass mit einem solchen Verständnis von Erziehung Zukunft etwas Geschlossenes sei, weil Zukunft nicht mehr als offen wahrgenommen und der nächsten Generation zur eigenen Gestaltung überlassen werde (Holfelder, 2019, S. 944). Dies gelte auch für die Vorstellung, dass Zukunft etwas komplett Kontingentes und Ungewisses sei, auf das man zukünftige Generationen durch flexibel einsetzbare Kompetenzen vorbereiten müsse. Beide Formen von Zukunftsvisionen seien problematisch, denn sie implizierten eine verkürzte Sicht auf das Individuum und auf Erziehung, in der die wirklichen Potenziale von Bildung für die gewünschte Gesellschaftstransformation verfehlt würden (Holfelder, 2019, S. 950).

Als Konsequenz dieser verschiedenen Konfliktlinien fordern kritische Stimmen neue, andere, emanzipatorische und pluralistische Formen von Bildung und Erziehung, die frei von vorbestimmten gesellschaftlichen und damit verbundenen pädagogischen Zielsetzungen sind und anstelle dessen verstärkt Möglichkeiten zur Selbstbestimmung, zur Entwicklung kritischen Denkens, zur pluralistischen Sinnfindung, Kommunikation und Kooperation und zur Teilhabe an demokratischen Dialogen und Prozessen der Entscheidungsfindung bieten (Öhman, 2007; Wals, 2010; Rowley, 2004). In dieser pluralistischen Vision von Erziehung und Bildung für eine offene Zukunft wird Erziehung als eine Plattform für die Entwicklung kritischer und neuer Wege des Denkens betrachtet, für die Schaffung von Bedingungen und Möglichkeiten für die Entwicklung alternativer Formen von Zukunft, für die Transformation etablierter Denkweisen und die Schaffung neuer Formen des gesellschaftlichen Zusammenlebens. Solche Visionen basieren auf den Ansprüchen an Bildung und Erziehung in einer demokratischen Gesellschaft und sie tragen auch dem Umstand Rechnung, dass die „richtigen“ Erziehungsziele für eine ungewisse und unvorhersehbare Zukunft selbst ungewiss sind. Sie tragen auch der Ungewissheit, Nicht-Kausalität und Unvorhersehbarkeit von Lern- und Bildungsprozessen Rechnung. 
Die Diskussionen zu den inhärenten Problemen und Widersprüchen einer Nachhaltigkeitsbildung für eine ungewisse und offene Zukunft unterstreichen die Bedeutung und die Potenziale des Philosophierens mit Kindern und Jugendlichen als ein Unterrichtsprinzip und eine Pädagogik, in der Ungewissheit in verschiedener Hinsicht eine wichtige Rolle spielt. Das Philosophieren mit Kindern kann als ein Modell einer kritischen und demokratischen Pädagogik betrachtet werden, weil es einen demokratischen Denk- und Erfahrungsraum bietet, dessen Ergebnisse nicht vorbestimmt sind, und der darauf abzielt, bestehende Machtverhältnisse sowohl im gesellschaftlichen Leben als auch zwischen den Generationen in Frage zu stellen.

Die philosophische Forschungsgemeinschaft bereitet darauf vor, mit Ungewissheit angstfrei und konstruktiv umzugehen und im Raum des Offenen und Ungewissen kreativ neue Ideen zu entwickeln. Diese Offenheit und Ungewissheit ist zentral für den Anspruch einer demokratischen Erziehung und kritischen Pädagogik, weil sie prinzipiell auch die Möglichkeit einer Transformation von Gesellschaft miteinschließt (Haynes, 2016; Echeverria \& Hannam, 2017; Burgh, 2018; Michaud, 2020). Philosophische Gespräche bieten einen Raum für Meinungs-, Gedanken- und Redefreiheit. Sie sollen nicht für Demokratie oder für Zukunft als etwas durch vorausgesetzte Werte, Haltungen und Einstellungen bereits Vorbestimmtes erziehen, sondern als eine demokratische Praxis Bedingungen schaffen für neue demokratische Lebensformen deren Inhalte nicht vorbestimmt, sondern durchaus ungewiss und offen sind. In dieser Hinsicht hat das Philosophieren mit Kindern als ein Unterrichtsprinzip und als eine pädagogische Haltung viele Potenziale für die Gestaltung zukunftsoffener Bildungsprozesse, die es für verschiedene Bildungsbereiche zu erschließen gilt.

\section{Literaturverzeichnis}

Barnett, Ronald (2000). Supercomplexity and the Curriculum. Studies in Higher Education, 25(3), 255-265. https://doi.org/10.1080/713696156

Barnett, Ronald (2012). Learning for an unknown future. Higher Education Research \& Development, 31(1), 65-77. https://doi.org/10.1080/07294360.2012.642841

Beghetto, Ronald A. (2017). Inviting Uncertainty into the Classroom. Five strategies to help students respond well to uncertainty and foster complex problem - solving skills. Unleashing Problem Solvers, 75(2), 20-25.

Birch, Rosamund (2020). Discerning Hope: Intra-Actions of a Philosophy for Children Workshop and the Eco-Socially Just Potential of Practicing Hope. Journal of Philosophy of Education, 54(4), 975-987. https://doi.org/10.1111/1467-9752.12484

Birnbacher, Dieter (2016). Klimaethik. Nach uns die Sintflut? Stuttgart: Reclam.

Brüning, Barbara (2021). Klima + Ethik. Ethische und religiöse Grundfragen kontrovers und schülerzentriert. Berlin: Cornelsen.

Brüning, Barbara \& Nachtsheim, Daniel (2021). Klima. Krise. Kinder. Philosophieren über Nachhaltigkeit und Fridays for Future. Weinheim: Beltz Juventa.

Burgh, Gilbert (2018). The need for philosophy in promoting democracy: A case for philosophy in the curriculum. Journal of Philosophy in Schools, 5(1), 38-58. https://doi.org/10.21913//PS.v5i1.1483

D'Agnese, Vasco (2018). Courage, Uncertainty and Imagination in Deweyan Work: Challenging the Neo-Liberal Educational Agenda. Journal of Philosophy of Education 52(2), 316-329. https://doi.org/$\underline{10.1111 / 1467-9752.12288}$

Davies, Katherine (2012). How reflective practice can enhance learning for sustainability. In Arjen E. J. Wals \& Peter B. Corcoran (Eds.), Learning for sustainability in times of accelerating change (pp. 283-296). Wageningen: Wageningen Academic Publishers.

De Haan, Gerhard; Kamp, Georg; Lerch, Achim; Martignon, Laura; Müller-Christ, Georg \& Nutzinger, Hans G. (2008). Nachhaltigkeit und Gerechtigkeit. Grundlagen und schulpraktische Konsequenzen. Berlin: Springer Verlag. 
Echeverria, Eugenio \& Hannam, Patricia (2017). The community of philosophical inquiry (P4C): A pedagogical proposal for advancing democracy. In Maughn R. Gregory; Joanna Haynes \& Karin Murris (Eds.), The Routledge International Handbook of Philosophy for Children (pp. 3-10). New York: Taylor \& Francis.

Fecho, Bob (2013). Globalization, localization, uncertainty and wobble: Implications for education. International Journal for Dialogical Science, 7, 115-128.

Funston, James (2017). Toward a Critical Philosophy for Children. PSU McNair Scholars Online Journal, 11(1), 1-17. https://doi.org/10.15760/mcnair.2017.05

Gebhard, Ulrich \& Michalik, Kerstin (2017). „Ist Tugend lehrbar?“ Zwischen Werteerziehung und kritischer Urteilsbildung. In Thomas Pyhel, Alexander Bittner, Anna-Katharina Klauer \& Vera Bischoff (Hg.), Umweltethik für Kinder. Impulse für die Nachhaltigkeitsbildung (S. 79-92). München: oekom-Verlag.

Gordon, Mordechai (2006). Welcoming Confusion, Embracing Uncertainty. Education Teacher Candidates in an Age of Certitude. Paideusis, 15(2), 15-25.

Hall, Brendan (2006). Teaching and learning uncertainty in science: the case of climate change. Planet, 17(1), 48-49. https://doi.org/10.11120/plan.2006.00170048

Hayden, Matthew J. (2012). Cosmopolitan Education and Moral Education: Forging Moral Beings under Condition of Global Uncertainty. PhD Thesis. New York: Columbia University.

Haynes, Joanna (2016). Philosophy with Children: An Imaginative Democratic Practice. In Helen E. Lees \& Nel Noddings (Eds.), The Palgrave International Handbook of Alternative Education (pp. 273-287). London: Palgrave Macmillan.

Helsper, Werner; Hörster, Reinhard \& Kade, Jochen (Hg.) (2003). Ungewissheit. Pädagogische Felder im Modernisierungsprozess. Weilerswist: Velbrück.

Holfelder, Anne-Katrin (2019). Towards a sustainable future with education? Sustainability Science, 14, 943-952. https://doi.org/10.1007/s11625-019-00682-z

Jordan, Michelle E. \& McDaniel, Reuben R. (2014). Managing Uncertainty During Collaborative Problem Solving in Elementary School Teams: The Role of Peer Influence in Robotics Engineering Activity. Journal of the Learning Sciences, 23(4), 490-536. https://doi.org/10.1080/10508406.2014.896254

Kahn, Richard V. (2010). Critical Pedagogy, Ecoliteracy, \& Planetary Crisis. The Ecopedagogy movement. New York: Peter Lang Publishing.

Kant, Immanuel (1968). Logik, Physische Geographie, Pädagogik. Berlin: Walter de Gruyter.

Kavanagh, Anne M.; Waldron, Fionnuala \& Mallon, Benjamin (Eds.) (2021). Teaching for Social Justice and Sustainable Development Across the Primary Curriculum. New York: Routledge.

Kohan, Walter O. (2018). Paulo Freire and Philosophy for Children: A Critical Dialogue. Studies in Philosophy and Education, 37(18), 615-629. https://doi.org/10.1007/s11217-018-9613-8

Kopnina, Helen (2012). Education for Sustainable Development (ESD): The turn away from 'environment' in environmental education? Environmental Education Research, 18(5), 699-717. https://doi.org/$\underline{10.1080 / 13504622.2012 .658028}$

Kopnina, Helen (2014). Education for sustainable development (ESD): Exploring anthropocentric-ecocentric values in children through vignettes. Studies in Educational Evaluation, 41, 124-132. https://doi.org/10.1016/j.stueduc.2013.12.004

Kopnina, Helen (2020). Education for the future? Critical evaluation of education for sustainable development goals. The Journal of Environmental Education, 51(1), 1-12. https://doi.org/10.1080/00958964.$\underline{2019.1710444}$

Kopnina, Helen \& Cherniak, Brett (2015). Cultivating a Value for Non-Human Interests through the Convergence of Animal Welfare, Animal Rights, and Deep Ecology in Environmental Education. Education Sciences, 5(4), 363-379. https://doi.org/10.3390/educsci5040363

Kopnina, Helen \& Meijers, Frans (2014). Education for sustainable development (EDS). Exploring theoretical and practical challenges. International Journal of Sustainability in Higher Education, 15(2), 188- 


\section{7. https://doi.org/10.1108/IJSHE-07-2012-0059}

Künzli David, Christine; Buchs, Christoph \& Wüst, Letizia (2015). Die Bedeutung des Philosophierens mit Kindern in einer Bildung für eine Nachhaltige Entwicklung. In Hans-Joachim Fischer, Hartmut Giest \& Kerstin Michalik (Hg.), Bildung im und durch Sachunterricht (S. 115-120). Bad Heilbrunn: Julius Klinkhart.

Lambrechts, Wim \& Hindson, James (Eds.) (2016). Research and Innovation in Education for Sustainable Development. Wien: Environment and School Initiatives.

Lipman, Matthew; Sharp, Ann Margaret \& Oscanyan, Frederick S. (1980). Philosophy in the Classroom (2nd Edition). Philadelphia: Temple University Press.

Lipman, Matthew (2003). Thinking in education (2nd Edition). Cambridge: Cambridge University Press. Lyle, Sue (2018). Education for Sustainable Development and Global Citizenship through Philosophical Enquiry: Principles and Practices. Analytic Teaching and Philosophical Practice, 39(1), 1-12.

MacDonald, Margaret \& Bowen, Warren (2016). Sharing Space with Other Animals: Early Childhood Education, Engaged Philosophical Inquiry, and Sustainability. Analytic Teaching and Philosophical Praxis, 37(1), 20-28.

MacDonald, Margaret; Bowen, Warren \& Hill, Cher M. (2017). Using engaged philosophical inquiry to deepen young children's understanding of environmental sustainability: Being, becoming and belonging. Journal of Philosophy in Schools, 4(1), 50-73.

Marcussen, Eirik H.; Weiss, Michael \& Helskog, Guro H. (2021). How Philosophizing the Dialogos Way Can Promote Education for Sustainable Development. In Teacher Education in the 21st CenturyEmerging Skills for a Changing World. Retrieved from https://www.intechopen.com/chapters/75268 [25.09.2021].

Meadows, Donella H.; Meadows, Dennis L.; Randers, Jørgen \& Behrens III, William W. (1972). The limits to growth. New York: Universe Books.

Michalik, Kerstin (2013). Philosophieren mit Kindern als Unterrichtsprinzip - Bildungstheoretische Begründungen und empirische Fundierungen. Pädagogische Rundschau, 67(6), 635-649.

Michalik, Kerstin (2016). Philosophische Gespräche im Unterricht. Bildungstheoretische Grundlagen und empirische Befunde. In Ludwig Duncker \& Bettina Uhlig (Hg.), Fragen - Kritik - Perspektiven. Theoretische Grundlagen des Philosophierens mit Kindern (S. 115-132). München: Kopaed Verlag.

Michalik, Kerstin (2019). Teacher and learner perspectives on Philosophical Discussion. Uncertainty as a Challenge and Opportunity. Childhood and Philosophy, 15, 125-144. https://doi.org/10.12957/childphilo.2019.42675

Michalik, Kerstin \& Schreier, Helmut (2017). Wie wäre es, einen Frosch zu küssen? Philosophieren mit Kindern im Grundschulunterricht (2. Auflage). Braunschweig: Westermann.

Michaud, Olivier (2020). What Kind of Citizen is Philosophy for Children Educating? What Kind of Citizen Should it be Educating? Philosophical Inquiry in Education, 27(1), 31-45.

Morehouse, Richard E. (2018). Caring thinking, education of emotions, and the community of inquiry: a psychological perspective. In Maughn R. Gregory \& Megan J. Laverty (Eds.), In Community of Inquiry with Ann Margaret Sharp. Childhood, Philosophy and Education (pp.197-208). London: Routledge.

Müller, Hans-Joachim unter Mitarbeit von Susanne Schubert (2011). Mit den Kleinen Großes denken. Mit Kindern über Nachhaltigkeitsfragen philosophieren - Ein Handbuch. Frankfurt am Main: Leuchtpol.

Murris, Karin S. (2008). Philosophy with Children, the Stingray and he Educative Value of Disequilibrium. Journal of Philosophy of Education, 42(3/4), 667-685. https://doi.org/10.1111/j.1467-9752.2008.$\underline{00640 . x}$

OECD (2005). Definition und Auswahl von Schlüsselkompetenzen. Abgerufen unter www.oecd.org/pisa/35693281.pdf [25.09.2021].

Öhman, Johan (2007). The Ethical Dimension of ESD - Navigating Between the Pitfalls of Indoctrination and Relativism. Retrieved from https://www.academia.edu/28199221/5 The Ethical Dimension of ESD Navigating Between the Pitfalls of Indoctrination and Relativism [25.09.2021]. 
O'Riordan, Nicola Jane (2016). Swimming against the tide: philosophy for children as a counter-cultural practice. Education 3-13, 44(6), 648-660.

Ott, Konrad (2010). Umweltethik zur Einführung. Hamburg: Junius Verlag.

Paseka, Angelika; Keller-Schneider, Manuela \& Combe, Arno (Hg.) (2018). Ungewissheit als Herausforderung für pädagogisches Handeln. Wiesbaden: Springer VS.

Prust, Christian \& Sukopp, Thomas (2017). Philosophieren mit Kindern im Sachunterricht mit dem Schwerpunkt „Nachhaltigkeit“: Möglichkeiten und Grenzen. In Martin Gröger, Mareike Janssen \& Jutta Wiesemann (Hg.), Nachhaltig Handeln lernen im Sachunterricht. Beitragsdokumentation zur Tagung am 5. Oktober 2016 an der Universität Siegen (S. 183-196). Siegen: universi.

Reder, Michael; Gösele, Andreas; Köhler, Lukas \& Wallacher, Johannes (2019). Umweltethik. Eine Einführung in globaler Perspektive. Stuttgart: Kohlhammer.

Roser, Dominic \& Seidel, Christian (2015). Ethik des Klimawandels. Eine Einführung (2. erweiterte Auflage). Darmstadt: WBG.

Rowley, Chris (2004). Philosophical Enquiry as an approach to Environmental Education. Place and Space Occasional Paper No 4, 87-91. Retrieved from https://www.geography.org.uk/download/ga eypprractionresearch4rowley.pdf [25. September 2021].

Rowley, Chris \& Lewis, Lizzy (2003). Thinking on the edge. Kendal: Living Earth.

Scholl, Rosie; Nichols, Kim \& Burgh, Gilbert (2014). Transforming pedagogy through philosophical inquiry. International Journal of Pedagogies and Learning, 9(3), 253-272.

Sharp, Ann Margaret (2018). The other dimension of caring thinking. In Maugh R. Gregory \& Megan J. Laverty (Eds.), In Community of Inquiry with Ann Margaret Sharp. Childhood, Philosophy and Education (pp. 209-214). London: Routledge.

Sukopp, Thomas (2020). Interculturality in Philosophy Education: Challenges and Prospects of Education for Sustainable Development in Primary Schools. In Martin Gröger, Christian Prust \& Alexandra Flügel (Hg.), Cultural Appropriation of Spaces and Things (pp. 113-134). Siegen: universi.

Tauritz, Rebekah L. (2012). How to handle knowledge uncertainty: learning and teaching in times of accelerating change. In Arjen E. J. Wals \& Peter B. Corcoran (Eds.), Learning for sustainability in times of accelerating change (pp. 299-316). Wageningen: Wageningen Academic Publishers.

Tauritz, Rebekah L. (2016). A pedagogy for uncertain times. In Wim Lambrechts \& James Hindson (Eds.), Research and Innovation in Education for Sustainable Development (pp. 90-105). Wien: Environment and School Initiatives.

Tauritz, Rebekah L. (2019). Certain you are not sure? An inquiry into pedagogical strategies for teaching children how to manage uncertain knowledge about sustainability challenges. Doctoral Thesis. The University of Edinburgh.

Tiedemann, Markus (2007). Schulung der Urteilskraft - Mit Kindern über Freiheit, Gerechtigkeit und Verantwortung philosophieren. In Dagmar Richter (Hg.), Politische Bildung von Anfang an. Demokratie-Lernen in der Grundschule. (S. 321-334): Schwalbach: Wochenschau Verlag.

UNESCO (2005). United Nations Decade of Education for Sustainable Development (2005-2014). In Framework for the International Implementation Scheme Volume 32. Paris: UNESCO.

UNESCO (2017). Education for Sustainable Development Goals. Learning Objectives. Paris: UNESCO.

Waldron, Fionnuala; Mallon, Benjamin \& Kavanagh, Anne M. (2021). Transforming pedagogy for a socially just and sustainable world. Concluding thoughts. In Anne M. Kavanagh, Fionnuala Waldron \& Benjamin Mallon (Eds.), Teaching for Social Justice and Sustainable Development Across the Primary Curriculum (pp. 231-235). New York: Routledge.

Wals, Arjen E. J. (2010). Between knowing what is right and knowing that it is wrong to tell others what is right: on relativism, uncertainty and democracy in environmental and sustainability education. Environmental Education Research, 16(1), 143-151.

Wals, Arjen E. J. \& Corcoran Peter B. (2012). Learning for Sustainability in times of accelerating change. Wageningen: Wageningen Academic Publishers. 
Weber, Barbara (2013). Philosophieren mit Kindern zum Thema Menschenrechte. Vernunft und Mitgefühl als Grundvoraussetzungen einer demokratischen Dialogkultur. Freiburg: Verlag Karl Alber. 